\title{
X-ray and optical observations of BL Lac objects: 3C 66A (B0219+428) and ON 325 (B1215+303)
}

\author{
M. Perri ${ }^{1,2}$, E. Massaro ${ }^{1,3}$, P. Giommi ${ }^{2}$, M. Capalbi ${ }^{2}$, R. Nesci ${ }^{1}$, G. Tagliaferri ${ }^{4}$, G. Ghisellini ${ }^{4}$, \\ M. Ravasio ${ }^{4}$, and H. R. Miller ${ }^{5}$
}

1 Dipartimento di Fisica, Università “La Sapienza”, Piazzale A. Moro 2, 00185 Roma, Italy
2 ASI Science Data Center, c/o ESA-ESRIN, via G. Galilei, 00044 Frascati, Italy
3 Istituto di Astrofisica Spaziale e Fisica Cosmica, CNR, via del Fosso del Cavaliere 100, 00133 Roma, Italy
4 Osservatorio Astronomico di Brera, via Bianchi 46, 22055 Merate, Italy
5 Department of Physics and Astronomy, Georgia State University, Atlanta GA, USA

Received 24 February 2003 / Accepted 10 June 2003

\begin{abstract}
We report the results of new observations of the two BL Lac objects 3C 66A and ON 325 performed with BeppoSAX and of nearly simultaneous photometric measurements in the bandpasses from $I$ to B. 3C 66A was observed in January 1999 and in July 2001: its X-ray flux was $F(2-10 \mathrm{keV})=2.0_{-0.5}^{+0.3} \times 10^{-12} \mathrm{erg} \mathrm{cm}^{-2} \mathrm{~s}^{-1}$ in January 1999 and $F(2-10 \mathrm{keV})=(2.6 \pm$ $0.6) \times 10^{-12} \mathrm{erg} \mathrm{cm}^{-2} \mathrm{~s}^{-1}$ in July 2001. In both observations the X-ray spectrum can be fitted by a single power law with an energy index $1.2 \pm 0.1$, steeper than that measured in the optical, equal to $0.86 \pm 0.05$. The BeppoSAX observation of ON 325 was performed in December 1998 when its X-ray flux was $F(2-10 \mathrm{keV})=8_{-4}^{+3} \times 10^{-13} \mathrm{erg} \mathrm{cm}^{-2} \mathrm{~s}^{-1}$. The X-ray spectrum cannot be fitted by a single power law, but it is found to be well represented by a broken power law with a break energy at around $4 \mathrm{keV}$ and energy indices equal to $1.48 \pm 0.12$ and $-0.57 \pm 0.63$. Both sources are characterised by a synchrotron-dominated emission at energies lower than a few $\mathrm{keV}$, with a sharp flattening toward higher energies due to the inverse Compton component. We obtained satisfactory modelling of the overall spectral energy distributions, from the optical to low energy X-rays, with logparabolic laws.
\end{abstract}

Key words. radiation mechanisms: non-thermal - galaxies: active - BL Lacertae objects: individual: 3C 66A, ON 325 X-rays: galaxies

\section{Introduction}

BL Lac objects have been classified into two main groups on the basis of their Spectral Energy Distribution (SED) by Padovani \& Giommi (1995): LBL (Low-energy peaked BL Lacs) if the maximum of the synchrotron (S) peak is in the range $10^{12}-10^{14} \mathrm{~Hz}$, and $\mathrm{HBL}$ (High-energy peaked BL Lacs) if it lies above $10^{16} \mathrm{~Hz}$. Compton scattered photons by relativistic electrons emitting the synchrotron radiation have energies in the $\mathrm{keV}$ and $\mathrm{GeV}-\mathrm{TeV}$ ranges for LBL and HBL sources, respectively. An open problem is the origin of the seed photons upscattered to high energies: they could either be the same radiated locally by synchrotron (SSC model, SynchroSelf Compton) or those originated in the surrounding regions (ERC model, External Radiation Compton) - see Sikora \& Madejski (2001) for a review. These two models predict different correlations between the optical and X-ray luminosities and different changes of the SED. In particular, in the SSC scenario, since the source of photons of the Inverse Compton (IC) emission are those produced through the synchrotron radiation, the $\mathrm{S}$ and IC components are strongly linked and the

Send offprint requests to: M. Perri, e-mail: perri@asdc . asi .it
IC emissivity has a quadratic dependence on the electron density. In the ERC models, instead, the $\mathrm{S}$ and IC components are less connected and the IC emission depends only linearly on the electron density. To investigate the possible behaviour we carried out nearly simultaneous optical and X-ray observations of a sample of BL Lacs. The best suited objects are those with the $\mathrm{S}$ peak in the near IR/optical band and the tail of this emission in the X-ray band where it mixes with the harder IC component. The BeppoSAX satellite (Boella et al. 1997a) was the best observatory for these observations because its Narrow Field Instruments (NFIs) cover a wide spectral energy range $(0.1-$ $200 \mathrm{keV}$ ) with a good sensitivity. Wide band X-ray observations combined with nearly simultaneous measurements in the optical are particularly useful to establish the spectral distribution around the synchrotron peak.

The selected sources are generally observed in active states detected either in the optical during intense monitoring programs or in the X-rays (see the review by Tagliaferri et al. 2002). The BL Lacs whose observations were triggered by optical flares were ON 231 (Tagliaferri et al. 2000), S5 0716+714 (Giommi et al. 1999; Tagliaferri et al. 2003), BL Lac (Ravasio et al. 2002), OJ 287 and MS 1458+22 (Massaro et al. 2003a). 
Table 1. BeppoSAX observation log and exposure times of 3C 66A and ON 325.

\begin{tabular}{cccccc}
\hline \hline Source & Date & Start UT & End UT & LECS Exp. (s) & MECS Exp. (s) \\
\hline 3C 66A & $1999 / 01 / 31$ & $02: 37$ & $17: 11$ & 8492 & 26441 \\
& $2001 / 07 / 29-30$ & $11: 48$ & $16: 23$ & 19840 & 50156 \\
ON 325 & $1998 / 12 / 23$ & $06: 26$ & $23: 59$ & 12545 & 31383 \\
\hline
\end{tabular}

For some of them we detected both the S and IC components. Two other sources, PKS 2005-489 (Tagliaferri et al. 2001) and Mkn 421 belonging to the HBL class, were observed because of an enhanced X-ray activity and showed only the $\mathrm{S}$ component well extended into the X-ray energy band. In this paper we present the results of BeppoSAX observations of the two BL Lac objects 3C 66A (B0219+428, J0222+4302) and ON 325 (B1215+303, J1217+3007).

The optical counterpart of the radio source 3C 66A was first identified by Wills \& Wills (1974); Miller et al. (1978) derived a redshift $z=0.444$ from a broad emission feature identified with a Mg II line. The classification as a BL Lac object is also supported by the high optical polarisation (16\%, Mead et al. 1990) and by the brightness variability with an amplitude of about 2 mag (Folsom et al. 1976; Miller \& McGimsey 1978).

The first X-ray observations of 3C 66A were obtained with the IPC instrument aboard the Einstein Observatory in 1979 and 1980 and are summarised by Maccagni et al. (1983). In July-August 1979 the source was very bright and reached a flux in the $0.2-4 \mathrm{keV}$ range of about $10^{-11} \mathrm{erg} \mathrm{cm}^{-2} \mathrm{~s}^{-1}$, more than one order of magnitude higher than that measured in February 1979 and July 1980. EXOSAT observed 3C 66A in 1986 on three occasions from January 6 to February 1.

The optical behaviour of $3 \mathrm{C} 66 \mathrm{~A}$ has been regularly monitored only since the end of 1994, mainly by the OJ 94 collaboration. This large database, described in Takalo et al. (1999), is directly accessible at the URL address http://www . astro.utu. fi/oj94/pub/pubdata. shtml. The EGRET source 3EG J0222+4253, whose error box contains 3C 66A, is indicated in the 3EG catalogue (Hartman et al. 1999) as its possible $\gamma$-ray counterpart. This high energy source is also positionally consistent with the millisecond pulsar PSR J0218+4232. Kuiper et al. (2000), on the basis of a Maximum Likelihood Ratio analysis of the $\gamma$-ray field, concluded that the emission at energies lower than $300 \mathrm{MeV}$ is essentially originated from the pulsar, while above $1 \mathrm{GeV}$ it is due to the BL Lac source.

ON 325 was first identified in the optical by Browne (1971) and classified as a BL Lac object because of its featureless continuum. A redshift $z=0.237$ was reported by Murphy et al. (1993), but more recently Bade et al. (1998) gave $z=0.130$. Zekl et al. (1981) reported optical variations larger than three magnitudes and a linear polarisation of $14 \%$ was measured by Wardle (1978). ON 325 was observed in the X-rays at various epochs with Einstein (December 1979), EXOSAT (December 1983, May 1985), ROSAT-PSPC (June 1991) and ASCA, but a detailed spectral study of its X-ray emission has not been published yet. Einstein data are reported by Worrall \& Wilkes (1990) and the results of the EXOSAT data observations are presented by Giommi et al. (1990) who reported flux changes in the energy band $0.05-2.0 \mathrm{keV}$ of a factor of about five. The spectral shape, however, was not determined because of the contamination in the ME instrument by a nearby source. Ueda et al. (2001) measured with ASCA a flux in the $2-10 \mathrm{keV}$ interval of $1.1 \times 10^{-12} \mathrm{erg} \mathrm{cm}^{-2} \mathrm{~s}^{-1}$.

\section{Observations and data reduction}

\subsection{X-ray data}

3C 66A was observed twice by BeppoSAX on January 31, 1999 for about $26 \mathrm{ks}$ and on July 29-30, 2001 for about $50 \mathrm{ks}$. ON 325 was observed on December 23, 1998 for about $31 \mathrm{ks}$.

Although on both occasions 3C 66A was not very bright in the X-rays, it was nevertheless very well detected in the LECS (Parmar et al. 1997) and MECS (Boella et al. 1997b) images. Also ON 325 was clearly detected by these two instruments. The presence of nearby confusing sources and a rather low count rate, did not allow us the use of PDS (Frontera et al. 1997) data and therefore the useful energy ranges were limited to about $10 \mathrm{keV}$. The journal of these pointings with the net exposure times for the two NFIs used is given in Table 1. Standard procedures and selection criteria were applied to the data to avoid the South Atlantic Anomaly, solar, bright Earth and particle contamination using the SAXDAS v. 2.0.0 package. Data analysis was performed using the software available in the XANADU Package (XIMAGE, XRONOS, XSPEC).

The images of ON 325 in the LECS and MECS showed a pointlike source well separated from the nearby Seyfert galaxy Mkn 766: the events for spectral analysis were selected within circular regions, centred at the source position and with radii of $4^{\prime}$. The event selection is more complex for $3 \mathrm{C} 66 \mathrm{~A}$ because of the nearby radiogalaxy $3 \mathrm{C} 66 \mathrm{~B}$ at an angular distance of only $\sim 6^{\prime}$. We therefore limited the extraction radius in the images of both instruments to $3^{\prime}$ to reduce the possible contamination from $3 \mathrm{C}$ 66B. Furthermore, we limited the useful energy range of the LECS to $0.6 \mathrm{keV}$ because the PSF becomes broader at low energies while for ON 325 we considered all the data down to $0.1 \mathrm{keV}$. In the spectral analysis of both sources data were rebinned to assure a minimum number of 20 events in each bin. Background spectra were taken from the blank field archive.

In the spectral fitting of X-ray data we must consider the low energy absorption due to the interstellar gas. The galactic column densities cannot be derived directly from X-ray data because of the rather low statistics and also because the simple power law cannot be a good model being the spectrum in 
Table 2. Photometric magnitudes of 3C 66A and ON 325.

\begin{tabular}{ccccccc}
\hline \hline Source & Date & UT & $B$ & $V$ & $R_{\mathrm{C}}$ & $I_{\mathrm{C}}$ \\
\hline 3C 66A & $1999 / 02 / 01$ & & & & $14.30(.03)$ & \\
& $2001 / 07 / 29$ & $01: 05$ & $14.97(.02)$ & $14.52(.03)$ & $14.09(.02)$ & $13.53(.02)$ \\
ON 325 & $1998 / 12 / 23$ & $00: 35$ & $15.56(.03)$ & $15.10(.03)$ & $14.65(.03)$ & $14.17(.03)$ \\
\hline
\end{tabular}

this range a combination of a steep $\mathrm{S}$ component with a flatter IC component. We adopted, therefore, the $N_{\mathrm{H}}$ value derived from the $21 \mathrm{~cm}$ measurements (Dickey \& Lockman 1990) of $N_{\mathrm{H}}=8.98 \times 10^{20} \mathrm{~cm}^{-2}$ for $3 \mathrm{C} 66 \mathrm{~A}$ and $N_{\mathrm{H}}=1.69 \times 10^{20} \mathrm{~cm}^{-2}$ for $\mathrm{ON} 325$. The latter is well in agreement with the literature estimates of the $A_{V}$ value, equal to 0.08 and the $N_{\mathrm{H}}$ vs. $E(B-V)$ relation given by Massaro et al. (2000), calibrated with the Crab nebula spectrum for Solar abundances. We used this relation also to derive the extinction for $3 \mathrm{C} 66 \mathrm{~A}$ and found $A_{V}=0.47$, a value larger than those given in the literature (see the NED database), which are around 0.30 . For the sake of consistency we adopted $A_{V}=0.47$ in the evaluation of the optical spectrum.

\subsection{Optical data}

Nearly simultaneous optical photometry of both sources was performed with the $0.5 \mathrm{~m}$ reflector of the Astronomical Station of Vallinfreda (Rome), equipped with a CCD camera and standard Johnson $B, V$ and Cousins $R, I$ bandpasses. For the January 1999 observation of 3C 66A only measurements in the $R$ band are available: the source was monitored for about five hours with the $0.40 \mathrm{~cm}$ reflector, located at the Hard Labor creek Observatory of Georgia State University, starting from February $1,2^{\mathrm{h}} 20^{\mathrm{m}}$ UT, nine hours after the end of the BeppoSAX pointing and during this time the source flux remained stable. Aperture photometry was made with IRAF-apphot using several reference stars in the same field of the sources. The $V, R, I$ magnitudes of these stars are given in Fiorucci \& Tosti (1996), while the $B$ values were taken from Gonzalez-Perez et al. (2001).

The magnitudes of both sources, measured during the BeppoSAX pointings, are given in Table 2.

\section{Results}

\section{1. $3 C 66 \mathrm{~A}$}

In January 1999 the unabsorbed X-ray flux of 3C 66A was $F(2-10 \mathrm{keV})=2.0_{-0.5}^{+0.3} \times 10^{-12} \mathrm{erg} \mathrm{cm}^{-2} \mathrm{~s}^{-1}$, and in July 2001 it was moderately higher and equal to $(2.6 \pm 0.6) \times$ $10^{-12} \mathrm{erg} \mathrm{cm}^{-2} \mathrm{~s}^{-1}$. The spectral analysis of both observations was performed using the data in the range $(0.6-2.0) \mathrm{keV}$ for the LECS and (1.65-9) keV for the MECS. A simple power law model gives good fits for these two data sets. The best fit energy indices $\left(F(E)=K E^{-\alpha}\right)$ were $\alpha=1.2 \pm 0.1\left(\chi_{\mathrm{r}}^{2}=0.88\right.$, 21 d.o.f.) in January 1999 and $\alpha=1.27 \pm 0.06\left(\chi_{\mathrm{r}}^{2}=0.95\right.$, 37 d.o.f.) in July 2001; here and throughout the paper the errors correspond to 1 standard deviation for one interesting parameter. The rather low statistics of both observations prevents the

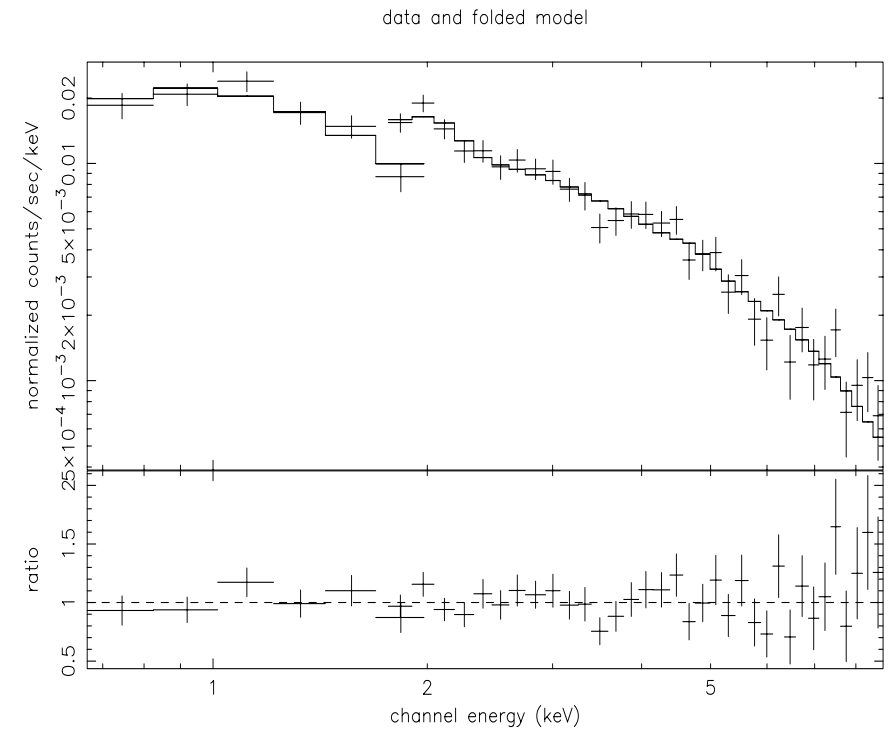

Fig. 1. Spectral best fit with a broken power law of the LECS and MECS data of the 2001, July 29-30 observation of 3C 66A.

possibility of a detailed study of the spectral distribution and a power law model is indeed well acceptable. Nevertheless, the SED of the July 2001 observation (see Fig. 2) shows an increasing trend toward the lower energies. This trend is clearly depending on the assumed Galactic $N_{\mathrm{H}}$ value and can disappear for smaller column density values. We modelled the spectrum with a broken power law:

$$
\begin{array}{rlrl}
F(E) & =K E^{-\alpha_{1}}, & & E \leq E_{\mathrm{b}} \\
& =K E_{\mathrm{b}}^{\alpha_{2}-\alpha_{1}} E^{-\alpha_{2}}, & E>E_{\mathrm{b}} .
\end{array}
$$

Of course we obtained again a very good fit $\left(\chi_{\mathrm{r}}^{2}=0.93\right.$, 35 d.o.f.) with a break energy $E_{\mathrm{b}}=2.4 \pm 0.7 \mathrm{keV}$ and nearly coincident spectral indices $\alpha_{1}=1.5 \pm 0.2$ and $\alpha_{2}=1.2 \pm 0.1$. This result is not statistically better than the single power law as indicated by the the $F$-test which gave a value of 1.46 with a significance of 0.246 . The plot of the broken power law best fit spectrum, practically identical to that of the single power law, is shown in Fig. 1.

The same trend observed in the X-ray flux is also apparent in the $R$ data (Table 2) being 3C 66A about 0.2 mag brighter in July 2001 than in January 1999. A power law fit to the optical points of July 2001, corrected for the reddening, gave an energy index $\alpha=0.86 \pm 0.05$. The corresponding $v F(v)$ values are shown with the X-ray data in the SEDs of Fig. 2. 


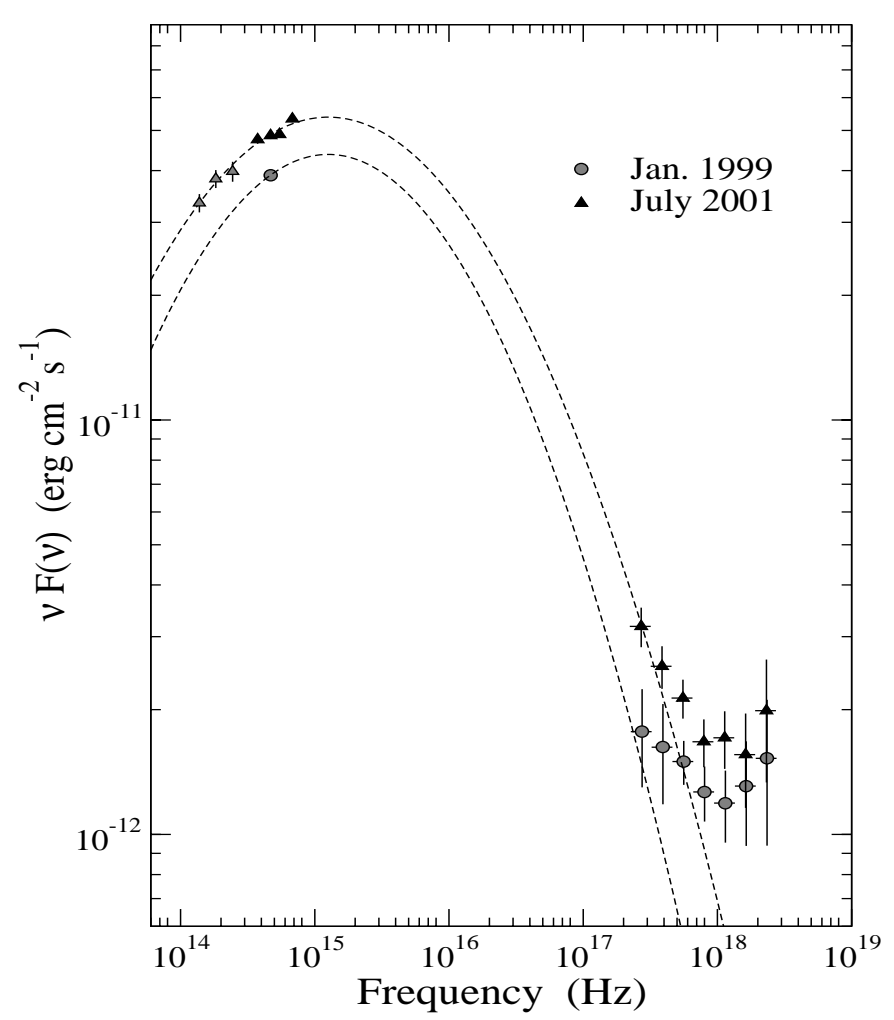

Fig. 2. The IR-optical to X-ray Spectral Energy Distribution of 3C 66A in the two BeppoSAX observations. Log-parabolic models of the synchrotron component are shown. $J, H$ and $K$ points (open triangles) are derived from our measurements taken one month after the BeppoSAX observation (Nesci et al. 2003) and have been reduced by 0.3 mag to match the optical fluxes as explained in Sect. 4 .

\subsection{ON 325}

The mean flux of ON 325 was about $8_{-4}^{+3} \times 10^{-13} \mathrm{erg} \mathrm{cm}^{-2} \mathrm{~s}^{-1}$, a factor of $\sim 3$ weaker than $3 \mathrm{C} 66 \mathrm{~A}$. Its X-ray spectrum cannot be well fitted by a single power law: the energy index was equal to $1.26 \pm 0.11$ but the corresponding reduced $\chi^{2}$ was 1.67 with 18 d.o.f. In particular, the most relevant discrepancy was a clear excess at energies above $5 \mathrm{keV}$ clearly apparent in the residuals plotted in Fig. 3. A much better fit is obtained with a broken power law - Eq. (1) -, for which we obtained $\chi_{\mathrm{r}}^{2}=1.10$ (16 d.o.f.), confirmed also by the $F$-test which gave a value of 5.63 with a significance of 0.014 . The plot of the broken power law best fit spectrum with the residuals is shown in Fig. 4. The two power laws intersect at $E_{\mathrm{b}}=4.0 \pm 0.6 \mathrm{keV}$ and the energy indices are $\alpha_{1}=1.48 \pm 0.12$ and $\alpha_{2}=-0.57 \pm 0.63$. Although the latter spectral index is poorly determined, likely because of the small band and the low flux, it is significantly flatter than that below $4 \mathrm{keV}$. This spectral distribution is likely due to the appearance of the inverse Compton component as already observed in other BL Lac objects, like S5 0716+714 (Giommi et al. 1999; Tagliaferri et al. 2003) and ON 231 (Tagliaferri et al. 2000). In Fig. 5 the SED of ON 325 derived from our optical and X-ray observations is shown.

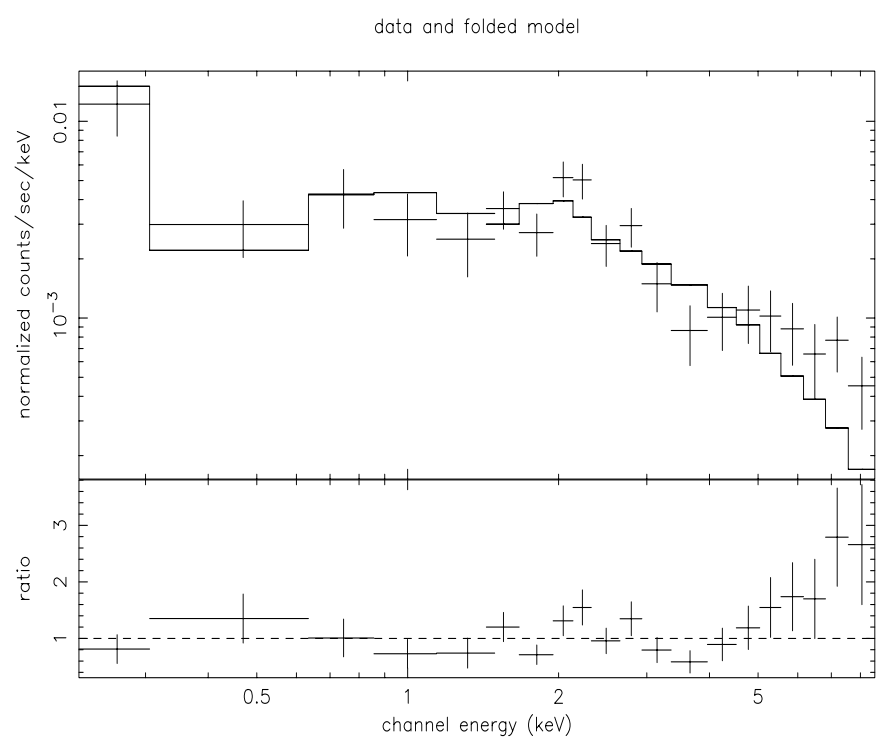

Fig. 3. Spectral best fit with a single power law of the LECS and MECS data of ON 325. Note the systematic excess at energies greater than about $5 \mathrm{keV}$.

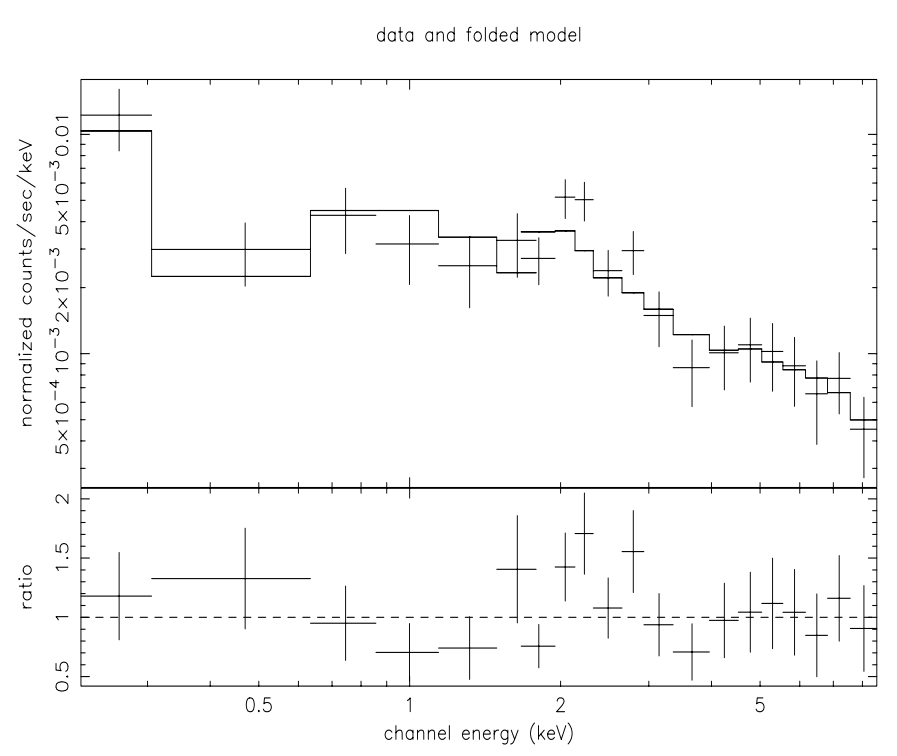

Fig. 4. Spectral best fit with a broken power law of the LECS and MECS data of ON 325.

\section{The Spectral Energy Distributions}

We used the optical and X-ray observations described above to derive more general information on the SEDs over the entire frequency range connecting the two bands. A common characteristics of 3C 66A and ON 325, like other BL Lac objects, is that the spectral slope in the optical is flatter than in the X-rays. This property is naturally explained by a synchrotron spectrum having a rather mild curvature, generally well described by a log-parabolic law which can be written in the following form to represent the SED:

$\log (v F(v))=\log \left(v_{\mathrm{p}} F\left(v_{\mathrm{p}}\right)\right)-b\left[\log \left(\frac{v}{v_{\mathrm{p}}}\right)\right]^{2}$ 


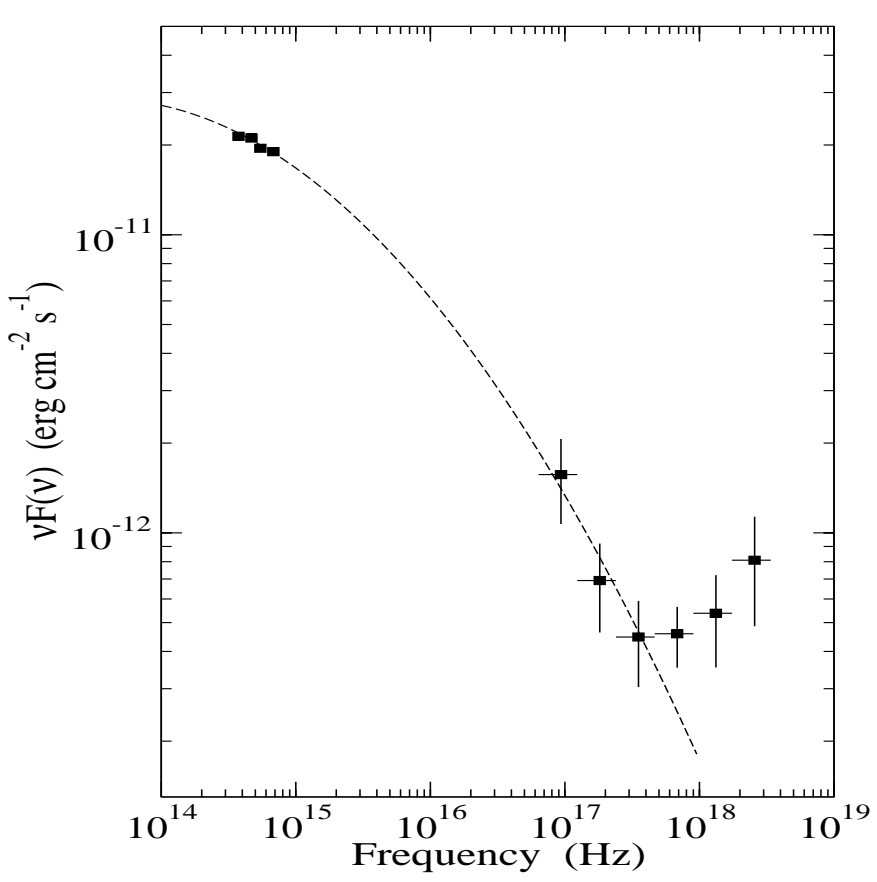

Fig. 5. The IR to X-ray SED of ON 325. The dotted line is a logparabolic interpolation of data.

where $v_{\mathrm{p}}$ is the peak frequency in the SED and $b$ a "curvature" parameter; it is easy to verify that the curvature radius at the parabola vertex is indeed equal to $1 /|2 b|$.

The success in describing the shape of the synchrotron spectra with a log-parabolic law was first pointed out by Landau et al. (1986) and more recently by Tanhiata et al. (2001) and Giommi et al. (2002). Massaro et al. (2002) proposed a simple interpretation for this law based on an energy dependent acceleration mechanism, described in more detail in Massaro et al. (2003b). Massaro et al. (2003a) used the log-parabolic model to describe the optical to X-ray synchrotron peak in the two BL Lac objects OJ 287 and MS 1458+22, also observed with BeppoSAX.

The same model was then applied to 3C 66A and ON 325. The SED of 3C 66A is shown in Fig. 2: the optical distribution is flat while that in the X-ray band is steep at low energies and flattens above $\sim 2 \mathrm{keV}$.

The flatness of the optical spectrum is confirmed also from the analysis of the OJ94 database by Hagen-Thorn et al. (2001) who report an average spectral index $0.92 \pm 0.12$ (computed with $\left.A_{V}=0.30\right)$. A log-parabolic SED has been determined in order to match the optical and the low energy X-ray data of the July 2001 observation. The resulting spectral parameters were $v_{\mathrm{p}}=1.26 \times 10^{15} \mathrm{~Hz}$ and $b=0.225$. A peak frequency in the near UV is more typical for the so called "intermediate" BL Lac objects rather than for LBL sources for which it lies in the near IR-optical range. The above $v_{\mathrm{p}}$ value is also in agreement with the SED plot given by Impey \& Neugebauer (1988). In Fig. 2 we also reported three photometric data in the $J, H$, and $K$ bands derived from our measurements obtained one month after the BeppoSAX observation (Nesci et al. 2003). These data were corrected by $0.3 \mathrm{mag}$ because the simultaneous $B V R I$ photometry showed that $3 \mathrm{C} 66 \mathrm{~A}$ was brighter by this amount than in July, but the colour indices were unchanged. The points correspond quite well to the low energy extrapolation of the log-parabolic spectrum and confirm, therefore, that this law gives a satisfactory model of the synchrotron peak over a frequency interval more than three orders of magnitude wide. For the observation of January 1999 we have only a value in the $R$ band and consequently the spectral slope in the optical is unknown: we assumed, therefore, the same $v_{\mathrm{p}}$ while $b$ resulted slightly higher and equal to 0.27 .

It is important to recall that the actual spectral shape depends upon some systematic effects which cannot be determined by our measurements. Assuming, for instance, a lower column density, equal to $6 \times 10^{20} \mathrm{~cm}^{-2}$, the X-ray flux at low energies is reduced as well as the reddening corrections. Another important systematic effect in the IR-optical band arises from the zero magnitude fluxes. The values given by Elvis et al. (1994), computed for a power law with an energy index of 1.0 and differing from those of Mead et al. (1990) less than 10\%, give spectral slope in the optical systematically steeper by about 0.3 . For $3 \mathrm{C} 66 \mathrm{~A}$ this implies that the peak frequency moves from the UV to the near IR. In the present analysis we take the values of Mead et al. (1990) for consistency with previous papers.

Although not statistically compelling, the most natural explanation of these data is that the X-ray emission of $3 \mathrm{C} 66 \mathrm{~A}$ is likely due to both the $\mathrm{S}$ and IC components: the former is only marginally detectable at energies below about $2 \mathrm{keV}$ while the other is responsible of the spectral flatness observed up to $\sim 10 \mathrm{keV}$.

In Fig. 5 we plotted the SED derived from our optical and X-ray observations of ON 325. In this case the detection of the synchrotron and inverse Compton components is better established. The optical spectrum has a best fit energy index of $1.22 \pm 0.06$ flatter than that in the low energy X-rays. A log-parabolic model gives a good description of the overall shape of the synchrotron peak. The model parameters have been chosen in order to fit the optical spectrum and to match the low energy X-ray points; their values are $v_{\mathrm{p}}=3.74 \times 10^{13} \mathrm{~Hz}$ and $b=0.113$.

\section{Discussion}

The results of BeppoSAX observations of these two BL Lac objects provide further evidence for the two component model of the SED and, in particular, give useful information on the energy distribution of the synchrotron component. We have already observed for other sources of the same class, like OJ 287 and MS 1458+22 (Massaro et al. 2003a), that the curved spectrum of the synchrotron component can be rather well described by a log-parabolic law. LBL sources are typically characterized by optical spectral indices around $0.8-1.3$ and the single power law extrapolation in the X-ray range gives a flux much higher than the observed ones. The log-parabolic model is one of the simplest ways to connect the IR-optical and X-ray emissions with a mild curved spectrum. The main parameter characterising this law is the "curvature" parameter $b$. We found that for 3C 66A this parameter has a value close to 0.23 whereas for ON 325 it is around 0.11 . 


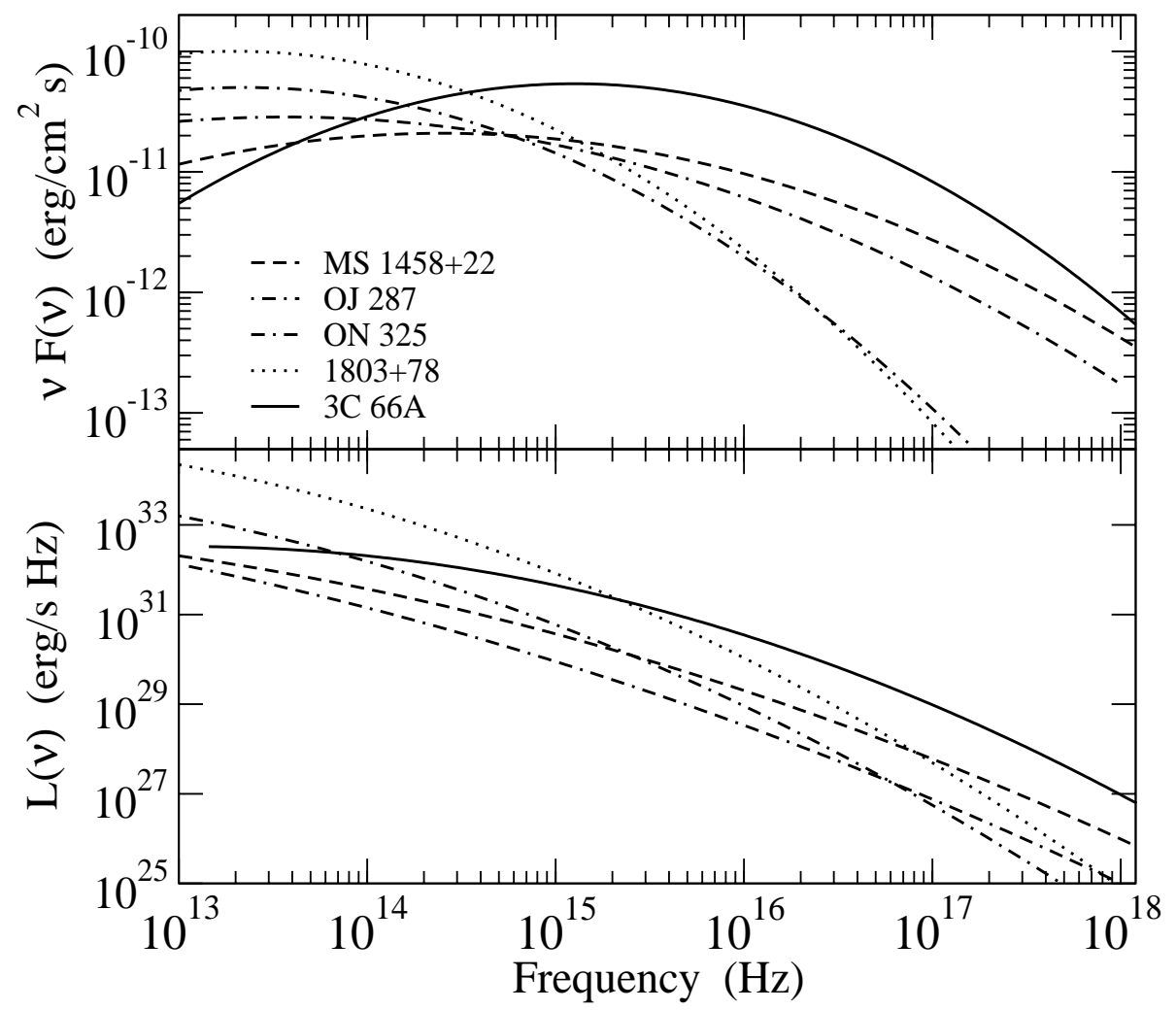

Fig. 6. Comparison between the Spectral Energy Distributions of the synchrotron component in some BL Lac objects measured in the observer (upper panel) and in the source frames (lower panel). The latter has been computed for an isotropic emission. All the distributions are modelled by means of log-parabolic laws: those for OJ 287 and MS 1458+22 are taken from Massaro et al. (2003a) and that for S5 1803+78, corresponding to its highest optical brightness, is taken from Massaro (2002).

Another advantage of this model is the possibility to integrate it analytically over the entire frequency range and to estimate the bolometric flux of the synchrotron component:

$F_{\mathrm{S}}=\sqrt{\pi \ln 10} \frac{v_{\mathrm{p}} F\left(v_{\mathrm{p}}\right)}{\sqrt{b}}=2.70 \frac{v_{\mathrm{p}} F\left(v_{\mathrm{p}}\right)}{\sqrt{b}}$.

In our cases we obtain $F_{\mathrm{S}} \simeq 3 \times 10^{-10} \mathrm{erg} \mathrm{cm}^{-2} \mathrm{~s}^{-1}$ for $3 \mathrm{C} 66 \mathrm{~A}$ and $F_{\mathrm{S}} \simeq 2.3 \times 10^{-10} \mathrm{erg} \mathrm{cm}^{-2} \mathrm{~s}^{-1}$ for ON 325. The corresponding apparent (not corrected for the beaming factor) luminosities can be computed from the luminosity distance

$d_{\mathrm{L}}=\left(c / H_{0}\right) z[1+z /(z+2+2 \sqrt{1+z})]$

for $q_{0}=0.5$. With the Hubble constant $H_{0}=65 \mathrm{~km} \mathrm{~s}^{-1} \mathrm{Mpc}^{-1}$ we derive $d_{\mathrm{L}}=2.2 \times 10^{3} \mathrm{Mpc}$ for $3 \mathrm{C} 66 \mathrm{~A}$ and $d_{\mathrm{L}}=6.2 \times$ $10^{2} \mathrm{Mpc}$ for ON 325, which imply (isotropic) luminosities of $\sim 1.8 \times 10^{47} \mathrm{erg} \mathrm{s}^{-1}$ and of $\sim 1.0 \times 10^{46} \mathrm{erg} \mathrm{s}^{-1}$, respectively.

It is interesting to compare the SEDs of some objects in the observer and in the source frames. In the upper panel of Fig. 6 we plot the observed log-parabolic distributions of five BL Lac objects. The observed X-ray flux distributions of three of them, namely $3 \mathrm{C} 66 \mathrm{~A}$, MS $1458+22$ and $\mathrm{ON} 325$, is characterized by the presence of a rather flat component (likely inverse Compton scattered photons) and, at energies below $\sim 3 \mathrm{keV}$ by a moderate excess due to the high energy tail of the synchrotron component. The other two, OJ 287 and S5 1803+78, showed in the BeppoSAX observations (Massaro et al. 2002, 2003a) only the IC component. In particular, the SED of S5 $1803+78$ plotted in
Fig. 6 corresponds to the most luminous optical state observed for this source (Nesci et al. 2002), but it was also observed a factor of about 15 fainter. Notice that the spectral curvature is similar for all the five sources: the values of the parameter $b$ lie in the rather narrow range from $\sim 0.11$ to $\sim 0.24$. Greater values, $b \simeq 0.35$, have been measured with BeppoSAX for the HBL source Mrk 421 in 1997-1999 (Massaro et al. 2003b).

The luminosity spectral distributions in the sources' frame are plotted in the lower panel of Fig. 6. Because of the different redshifts we see that the luminosities are scattered over two orders of magnitude, but this scatter can be reduced to a factor of $\sim 10$ if one considers that the luminosity of S5 $1803+78$ corresponds to a very bright state. The typical apparent (beamed) luminosities of these sources are then in the interval between $7 \times 10^{45}$ and $10^{46} \mathrm{erg} \mathrm{s}^{-1}$. The spread of the synchrotron peak frequencies is from $\sim 3 \times 10^{13}$ to $\sim 2 \times 10^{15} \mathrm{~Hz}$. To evaluate the actual energy outputs from these sources it is necessary to take into account the (unknown) beaming factors, which are usually assumed of the order of 10 . The similarities in the luminosity distributions suggests that also the spread of the beaming factor values cannot be large.

The log-parabolic law is not only a useful analytical tool to describe a curved SED, but it also allows a direct physical interpretation of the parameters. This type of spectra is naturally obtained from statistical acceleration mechanisms when the probability for a particle to remain inside the acceleration region is assumed to decrease with the energy of the particle 
itself. It is well known that a power law spectrum is obtained when the energy gain of a particle at the step $k, \gamma_{k}=\varepsilon \gamma_{k-1}$ ( $\gamma$ being the Lorentz factor of the electrons), and the probability $p$ that a particle has an energy gain $\varepsilon$ are both constant:

$N(>\gamma)=N_{0}\left(\gamma / \gamma_{0}\right)^{\log p / \log \varepsilon}$.

With the assumption that the acceleration probability decreases with the electron energy, and that this relation is given by a power law:

$p(\gamma)=g / \gamma^{q}$,

(with $g$ and $q$ positive constants), which follows, for instance, when the probability of a particle to be diffused against the shock front depends upon its radius of gyration in the ambient magnetic field, it is possible to find that the energy integral distribution of accelerated particles follows a log-parabolic law - see Massaro et al. (2003b) for the mathematical details -:

$N(>\gamma)=N_{0}\left(\gamma / \gamma_{0}\right)^{-s+r \log \left(\gamma / \gamma_{0}\right)}$,

where $r$ and $s$ depend upon the three acceleration parameters $g, q$ and $\varepsilon$. The differential distribution is not exactly a $\log$-parabola, but differs from this curve very little. It is easy to verify, at least in the $\delta$-approximation, that the synchrotron radiation from an electron population with this distribution is again a log-parabola and the relation between the curvature parameters of the particle and radiation spectra is:

$b=r / 4$.

A flare of the $\mathrm{S}$ component extending into the $\mathrm{X}$-ray range can be originated either by an increase of $v_{\mathrm{p}}$ and/or $F\left(v_{\mathrm{p}}\right)$, or by a change of $r$, and consequently of the curvature parameter $b$. Electrons emitting synchrotron X-rays have quite short radiative lifetimes and therefore the acceleration mechanisms must be active in the course of the flare. In this case one could expect that the observed spectral curvature is more related to the acceleration process rather than to the radiative cooling.

The last important topic is the relation between the $\mathrm{S}$ and the high energy emission. As stated in the Introduction, the EGRET source 3EG J0222+4253 has been indicated as the $\gamma$-ray counterpart of 3C 66A (Mukherjee et al. 1997; Hartmann et al. 1999). Lin et al. (1999) report a mean flux (>100 MeV) of $1.9 \pm 0.3$ phot $\mathrm{cm}^{-2} \mathrm{~s}^{-1}$ and a photon spectral index of $2.01 \pm$ 0.14 . These values have been questioned by Kuiper et al. (2000), who associated the flux at energies below $\sim 1 \mathrm{GeV}$ with the near millisecond pulsar PSR J0218+4232. In Fig. 7 we plotted the SED of 3C 66A from the near IR to the $\mathrm{GeV}$ frequencies as resulting from these $\gamma$-ray data and our measurements of 2001. We tried also to plot some possible spectral distributions of the IC component (dotted and dash-dotted lines), assumed again to be modelled by a log-parabolic law although there is no clear observational evidence for it. Because the data are not simultaneous, the $\gamma$-ray distributions were not computed by means of SSC or ERC models, but they have been drawn to match approximately the spectrum given by Lin et al. (1999). Note that the excess of the data at around $10^{18} \mathrm{~Hz}$ is only apparent since the IC contribution has not been added to the high energy tail of S emission. To reproduce with only

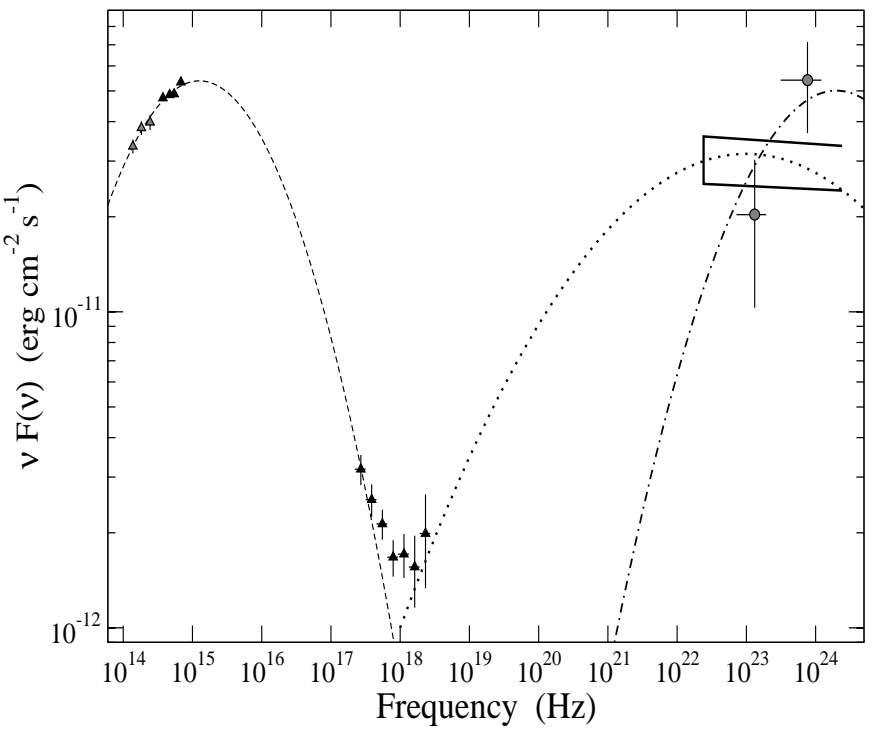

Fig. 7. The SED of 3C 66A from the near IR to $\gamma$-rays, obtained by our 2001 measurements combined with non simultaneous EGRET data according the spectrum by Lin et al. (1999) (open box) or the flux estimates above $300 \mathrm{MeV}$ by Kuiper et al. (2000) (open circles). The dotted and dash-dotted lines represent possible IC components.

one component both the possible IC emission apparent in the $\mathrm{X}$-rays and at energies above $30 \mathrm{MeV}$, it is necessary to take a spectrum much less curved ( $b=0.06$, dotted line in Fig. 7) than the S component in 2001.

On the other hand, the rather sharp low energy cutoff required by $\gamma$-ray emission according to the fluxes given by Kuiper et al. (2000), cannot be easily reconciled with the X-ray points. A possible example of such a spectral distribution is shown by the dash-dotted line in Fig. 7. To match the X-ray points it would require either a power law low energy tail or the presence of an additional component, extending mainly from hard X-ray band to low energy $\gamma$ rays. If future observations will provide evidence for such a composite spectral distribution, 3C 66A could be similar to the intermediate BL Lac object S5 0716+714 (Tagliaferri et al. 2003) for which these authors proposed that a contribution to IC $\gamma$-ray emission is likely due to another population of seed photons, for instance those originated in a region external to the jet as in the ERC model.

A good strategy to distinguish among these possible explanations is to perform coordinated observational campaigns covering a very wide frequency interval, at least from IR to the $\mathrm{GeV}$ range, to monitor the flux changes of the various components. These sources, therefore, must be considered among the main targets of future $\gamma$-ray wide field space observatories, like AGILE and GLAST, which can provide rich data sets to be compared with simultaneous measurements in the IR-optical-UV and X-ray bands.

Acknowledgements. Part of this work was performed with the financial support Italian MIUR (Ministero dell' Istruzione Universitá e Ricerca) under the grant Cofin 2001/028773. We acknowledge also the support by the Italian Space Agency (ASI) in the framework of the BeppoSAX mission. 


\section{References}

Bade, W., Beckmann, W., \& Douglas, N. G. 1998, A\&A, 334, 459

Boella, G., Butler, R. C., Perola, G. C., et al. 1997a, A\&AS, 122, 299

Boella, G., Chiappetti, L., Conti, G., et al. 1997b, A\&AS, 122, 327

Browne, I. W. A. 1971, Nature 231, 515

Elvis, M., Wilkes, B. J., McDowell, J. C., et al. 1994, ApJS, 95, 1

Dickey, J. M., \& Lockman, F. J. 1990, ARA\&A, 28, 215

Fiorucci, M., \& Tosti, G. 1996, A\&AS, 116, 403

Folsom, G. H., Miller, H. R., Wingert, D. W., \& Williamon, R. M. 1976, AJ, 81, 298

Frontera, F., et al. 1997, A\&AS, 122, 357

Giommi, P., Barr, P., Garilli, B., et al. 1990, ApJ, 356, 432

Giommi, P., Massaro, E., Chiappetti, L., et al. 1999, A\&A, 351, 59

Giommi, P., et al. 2002, Proc. Blazar Astrophysics with BeppoSAX and Other Observatories, ed. P. Giommi, E. Massaro, \& G. G. C. Palumbo (ASI Special Publication), 63

Gonzalez-Perez, J. N., Kidger, M. R., \& Martin-Luis, F. 2001, AJ, 122,2055

Hagen-Thorn, V. A., Hagen-Thorn, A. V., Jorstad, S. G., et al. 2001, Astrophys., 44, 283

Hartman, R. C., Bertsch, D. L., Bloom, S. D., et al. 1999, ApJS, 123, 79

Impey, C. D., \& Neugebauer, G. 1988, AJ, 98, 307

Kuiper, L., Hermsen, W., Verbunt, F., et al. 2000, A\&A, 359, 615

Landau, R., et al. 1986, ApJ, 348, 14

Lin, Y. C., Bertsch, D. L., Bloom, S. D., et al. 1999, ApJ, 525, 191

Maccagni, D., Maraschi, L., Tanzi, E. G., et al. 1983, ApJ, 273, 75

Massaro, E., Cusumano, G., et al. 2000, A\&A, 361, 695

Massaro, E. 2002, Proc. Blazar Astrophysics with BeppoSAX and Other Observatories, ed. P. Giommi, E. Massaro, \& G. G. C. Palumbo (ASI Special Publication), 3

Massaro, E., Giommi, P., Perri, M., et al. 2003a, A\&A, 399, 33
Massaro, E., Perri, M., Giommi, P., \& Nesci, R. 2003b, A\&A, submitted

Mead, A. R. G., Ballard, K. R., Brand, P. W. J. L., et al. 1990, A\&AS, 83,183

Miller, J. S., French, H. B., \& Hawley, S. A. 1978, in Pittsburg Conference on BL Lac Objects, ed. A. M. Wolfe, 176

Miller, H. R., \& McGimsey, B. Q. 1978, ApJ, 220, 19

Mukerjee, R., Berstch, D. L., Bloom, S. D., et al. 1997, ApJ, 490, 116

Murphy, D. W., Browne, I. W. A., \& Perley, R. A. 1993, MNRAS, 264, 298

Nesci, R., Massaro, E., Maesano, M., et al. 2002, AJ, 124, 53

Nesci, R., Sclavi, S., Maesano, M., et al. 2003, Mem. SAIt 74, 169

Padovani, P., \& Giommi, P. 1995, ApJ, 111, 222

Parmar, A. N., Martin, D. D. E., Bavdaz, M., et al. 1997, A\&AS, 122, 309

Ravasio, M., Tagliaferri, G., Ghisellini, G., et al. 2002, A\&A, 383, 763

Sambruna, R. M., Barr, P., Giommi, P., et al. 1994, ApJ, 434, 468

Sikora, M., \& Madejski, G. 2001, in High Energy Gamma-Ray Astronomy, ed. F. A. Aharonian, \& H. J. Volk, AIP Conf. Proc., 558,275

Tagliaferri, G., Ghisellini, G., Giommi, P., et al. 2000, A\&A, 354, 431

Tagliaferri, G., Ghisellini, G., Giommi, P., et al. 2000, A\&A, 368, 38

Tagliaferri, G., Ghisellini, G., Ravasio, M., et al. 2002, Proc. Blazar Astrophysics with BeppoSAX and Other Observatories, ed. P. Giommi, E. Massaro, \& G. G. C. Palumbo (ASI Special Publication), 11

Tagliaferri, G., Ravasio, M., Ghisellini, G., et al. 2003, A\&A, in press Takalo, L. O., Pursimo, T., Sillanpää, A., et al. 1999, Blaz. Data 1, n.6 Ueda, Y., Ishisaki, T., Takahashi, T., et al. 2001, ApJS, 133, 1

Wardle, J. F. C. 1978, Proc. Pittsburgh Conf. on BL Lac Objects, ed. A. M. Wolfe, 3

Wills, B. J., \& Wills, D. 1974, ApJ, 190, L269

Worrall, D. M., \& Wilkes, B. J. 1990, ApJ, 360, 396

Zekl, H., Klare, G., \& Appenzeller, I. 1981, A\&A, 103, 342 